\title{
Fabrication of an Antireflective Nanodome Array with High Packing Density for Photovoltaic Applications
}

\author{
Jiseok Lim, ${ }^{1}$ Sun-Mi Lee, ${ }^{2}$ Ho-Young Jang, ${ }^{2}$ and Seok-Min Kim ${ }^{2}$ \\ ${ }^{1}$ School of Mechanical Engineering, Yeungnam University, Gyeongsan 712-749, Republic of Korea \\ ${ }^{2}$ School of Mechanical Engineering, Chung-Ang University, Seoul 156-756, Republic of Korea \\ Correspondence should be addressed to Seok-Min Kim; smkim@cau.ac.kr
}

Received 22 May 2015; Revised 23 July 2015; Accepted 26 July 2015

Academic Editor: Xiaogang Han

Copyright (c) 2015 Jiseok Lim et al. This is an open access article distributed under the Creative Commons Attribution License, which permits unrestricted use, distribution, and reproduction in any medium, provided the original work is properly cited.

\begin{abstract}
This paper describes a nanofabrication method for an antireflective nanodome array with high packing density. The master for the close packed nanodome array was fabricated by industrially well-established photolithography and physical vapor deposition processes to realize uniform and defect-free nanostructure in large area. The nanodome array was formed on the surface of a glass plate by a nanoimprinting process using the replicated mold from the master with the aim of reducing the Fresnel reflection of the glass surface. The replication fidelity was verified using scanning electron microscopy and the nanodome structured glass substrate showed $\sim 3 \%$ increase in optical transmittance.
\end{abstract}

\section{Introduction}

Recently, the development of an antireflective surface to reduce the reflection that occurs at the interface between materials with different refractive indices had attracted research interest [1]. Antireflective surfaces have been applied to many applications, such as illumination, display, and imaging systems, as reflection can lead to performance issues in many optical and optoelectronic systems [2]. In particular, reflection is a very important issue for solar cell applications. Optical loss due to reflection at the optical interface of the solar cell is one of the most critical factors limiting the photovoltaic efficiency of solar cells [3]. To improve the conversion efficiency of solar cells, an antireflective surface is required.

Antireflection coatings (ARCs) made up of single- or multistacked layers with different refractive indices are widely used [4]. However, some of the limitations of these coating materials include optical bands and thermal mismatch between the film and substrate. As an alternative to ARCs, the use of antireflective nanostructures on optical interfaces has attracted intense interest [5]. The periodic subwavelengthpatterned surface enables continuous changes in refractive index that remove the optical interface [6]. Considerable research efforts have sought to develop antireflective nanopatterns on large optical surfaces for solar cell applications $[7,8]$.

Many studies evaluated the effect of the geometric shape of the nanostructure to the antireflection efficiency. Tapered shapes and pyramidal structures have been reported to be the most efficient design [9]. However, these nanostructure geometries have disadvantages in solar cell applications. To fabricate a sharp edge nanostructure on a large area at low cost is very challenging. Moreover, since the operation conditions of solar cell modules are relatively harsh, a linear tapered geometry can be easily damaged.

In this study, we propose an antireflective nanodome structure with high packing density and a cost-effective fabrication method using a nanoimprinting process. The nanoimprinting process is one of the most promising technologies for production of nanostructures at low cost [10, 11]. The most important element of the nanoimprinting process is a mold that has a negative shape of the final structure. Han et al. fabricated the mold for dome-shaped structured antireflection layer by polymer replication of electroformed master from the laser interference lithography [7] and Liu et al. fabricated the mold by electroforming of selfassembly nanosphere lithography [12]. The laser interference lithography and nanosphere lithography can be a promising 
method to fabricate master pattern for the mold of domeshaped antireflective nanostructure. However, this method has limitations to fabricate large area master pattern without any defects. In practical operation, it is difficult to fabricate uniform and defect-free dome-shaped nanostructure with more than $100 \mathrm{~mm}$ diameter size by the laser interference lithography or nanosphere lithography. In this study we fabricated replicated polymer mold from the dome-shaped master which was basically prepared by a photolithography and a physical vapor deposition process. The photolithography and physical vapor deposition process are industrially wellestablished techniques and can provide a large area uniform dome-shaped pattern (up to $300 \mathrm{~mm}$ diameter) without any defects. Finally, a nanodome array was replicated on a glass substrate using a UV imprinting process. As a result, the transmittance of the glass substrate was increased from 88$89 \%$ to $91-92 \%$ for a single-sided, patterned glass substrate in the spectral range of 350 to $850 \mathrm{~nm}$.

\section{Materials and Methods}

A polymeric mold was fabricated using UV imprinting and physical e-beam evaporation processes, and then the antireflective nanodome-patterned structure was replicated on a glass substrate using the UV imprinting process. An overview of the fabrication procedures is presented in Figure 1.

A silicon master pattern with $500 \mathrm{~nm}$ pitch, 0.5 duty, and $100 \mathrm{~nm}$ grating height was fabricated via $\mathrm{KrF}$ scanner photolithography and reactive ion etching processes. A bottom antireflection coating (BARC) with a thickness of $58 \mathrm{~nm}$ was spin-coated on an 8-inch wafer and a photoresist was spin-coated with $580 \mathrm{~nm}$ thickness. Photolithography was conducted using a $\mathrm{KrF}$ step and repeat scanner (NSRS203B, Nikon Co., Ltd., Japan), and the reactive ion etchings of the BARC layer and silicon substrate $110 \mathrm{~nm}$ in depth were performed using a poly etcher (TCP 9400DFM, Lam Research Co., Ltd.). The silicon master pattern was fabricated by the fab foundry service institute (National NanoFab Center, Daejeon, Korea). The fabricated silicon master wafer was subsequently treated with a self-assembled monolayer (SAM) coating to prevent adhesion of the cured polymer to the wafer. The SAM film was applied by dipping the wafer into a $2 \%$ solution of dimethyldichlorosilane dissolved in octamethylcyclooctasilane (Repel-Silane ES, GE Healthcare Co., Ltd., USA). The fabricated silicon master was replicated using the UV imprinting process on polyester (PET) film (SH34, SKC Co., Ltd., Korea) to prepare the polymeric master. The UV imprinting processes were performed using a UV curable urethane acrylate based photopolymer (UP088, SK Chemicals Co., Ltd., Korea) with a refractive index of 1.52 and a UV curing system (2000 Flood, Dymax Co. Ltd., USA). Since the polymeric master has a cylindrical nanocavity structure, it was replicated once more to produce a nanopillar pattern array (Figures 1(a) and 1(b)). In order to form a nanodome structure, $\mathrm{SiO}_{2}$ was deposited over the fabricated nanopillar pattern by e-beam evaporator (Modified SEE-7, Ultech Co., Ltd., Korea) with a deposition rate of $0.3 \AA / \mathrm{s}$ at a vacuum level of $6 \times 10^{-6}$ Torr, as shown in Figure 1(c).

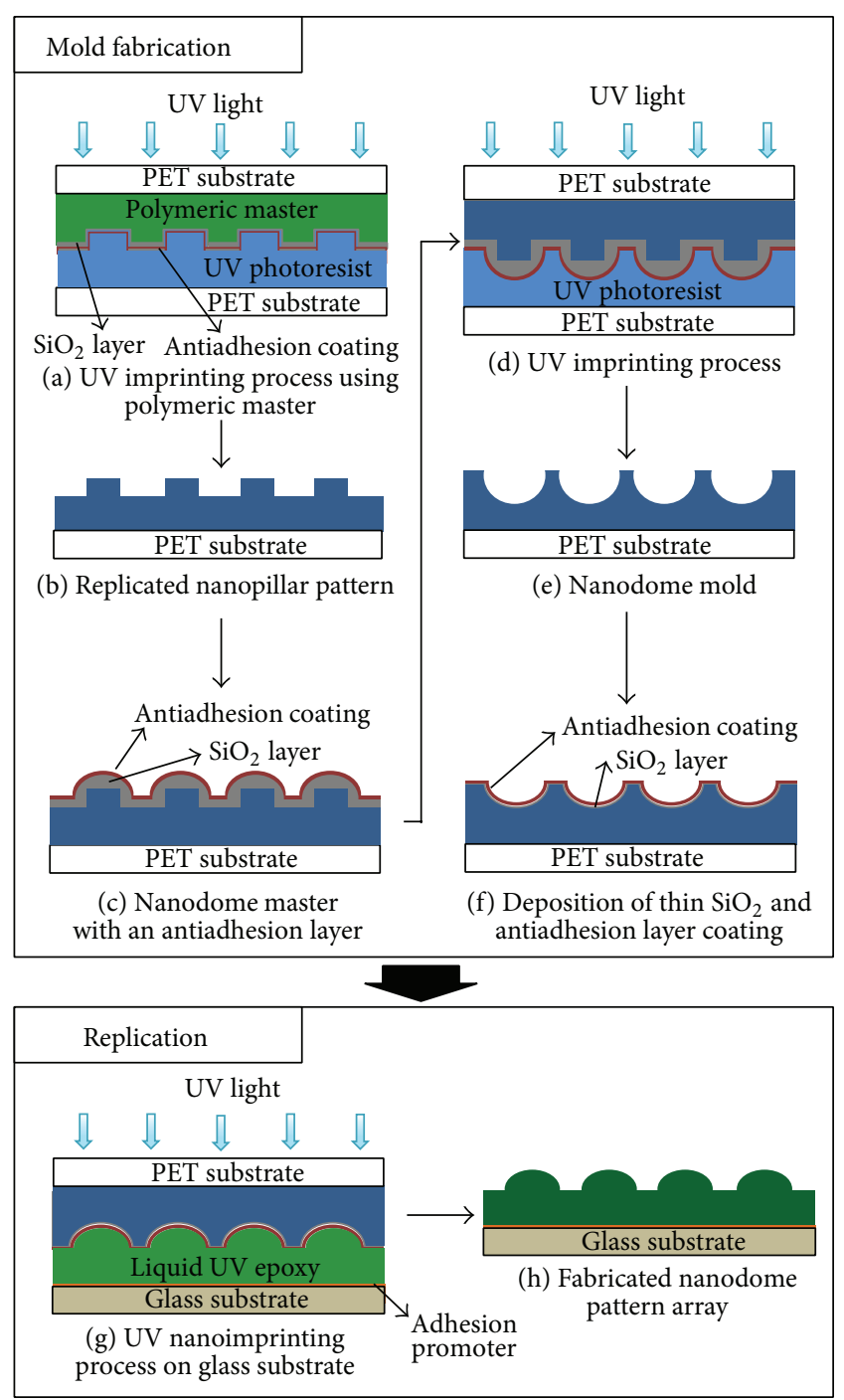

FIGURE 1: Schematic flow chart of nanodome pattern array fabrication.

The cylindrical pedestal structure evolves into a dome structure and the diameter of the dome structure increases with the deposition thickness of $\mathrm{SiO}_{2}$ [13]. To evaluate the effect of the $\mathrm{SiO}_{2}$ thickness on the geometric properties of the nanodome array, three different thicknesses of the $\mathrm{SiO}_{2}$ layer $(50,100$, and $150 \mathrm{~nm})$ were tested. The fabricated nanodome structure was subsequently replicated by UV imprinting to form a polymer mold with a nanodome cavity array. Finally, the nanodome structure was fabricated by transferring the pattern of the mold to a glass slide substrate (Duran Group Co., Ltd., Germany) using UV imprinting. To improve the adhesion between the polymer and glass substrate, an adhesion promoter (ZAP-1020, Chemoptics Inc., Korea) was deposited on the glass substrate, which form an optically transparent amine monolayer on the glass substrate.

An optical grade urethane acrylate based photopolymer (UP088, SK Chemicals Co., Ltd., Korea) was used for the series of UV imprinting processes, and the UV imprinting processes were performed using a UV curing system 


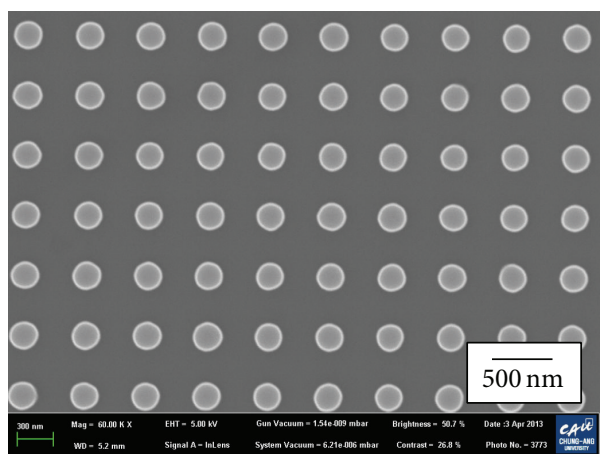

(a)

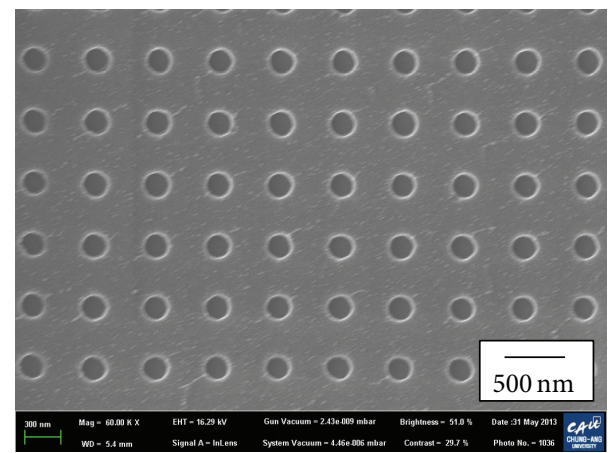

(b)
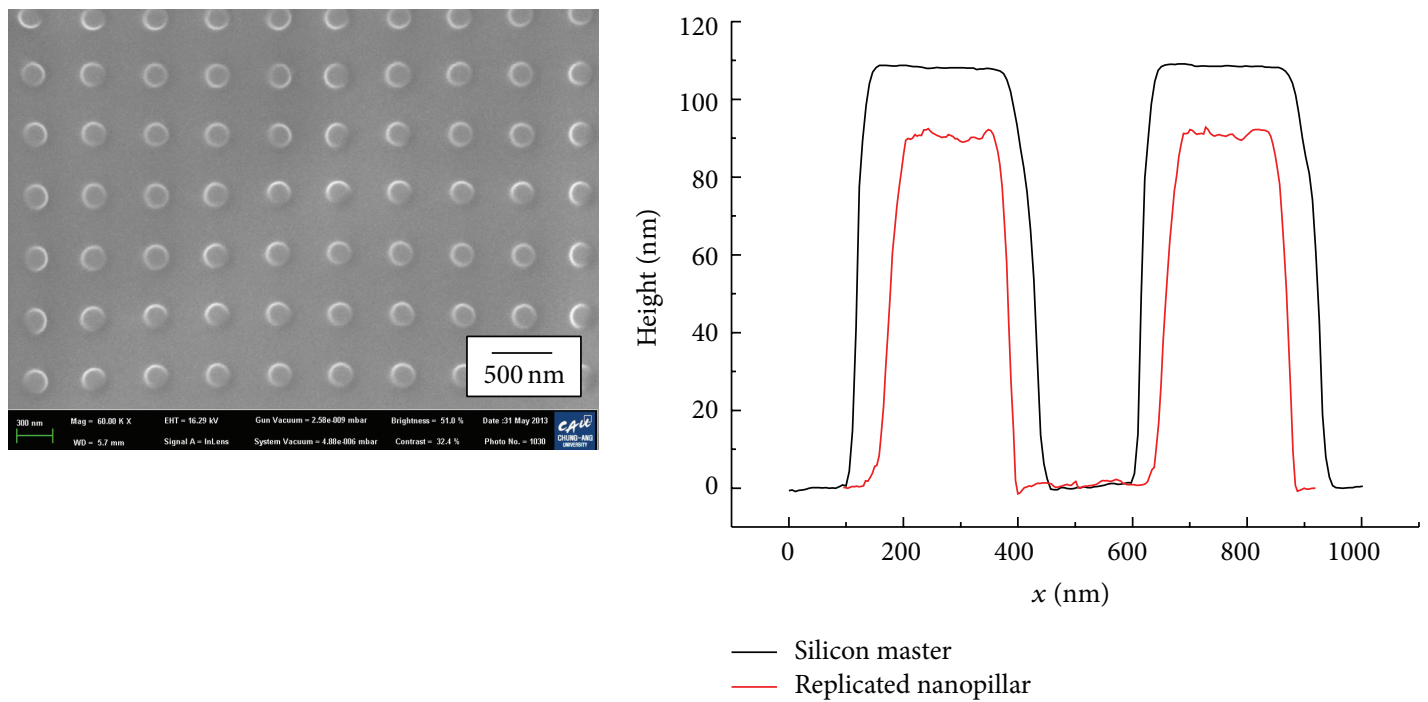

(c)

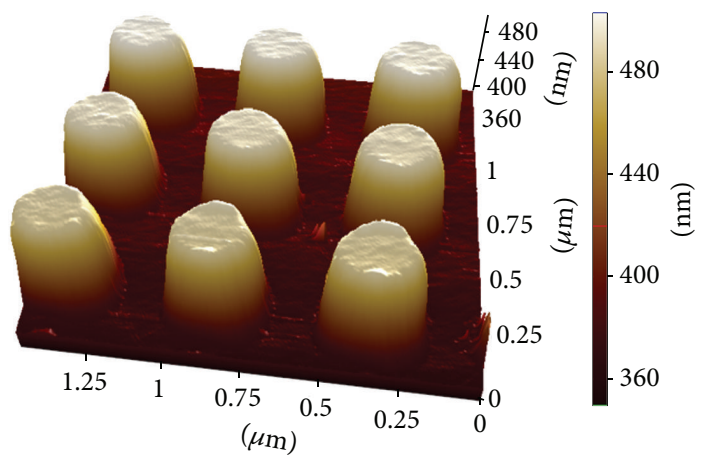

(e)

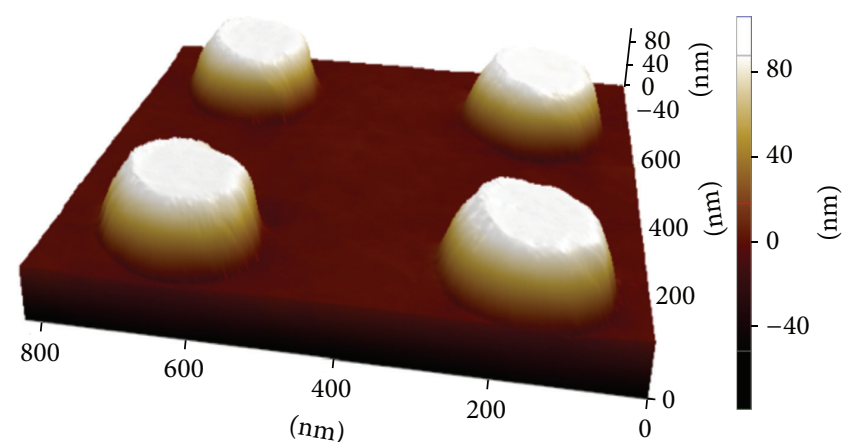

(f)

FIGURE 2: SEM of (a) silicon master, (b) polymeric master, and (c) replicated nanopillar pattern. (d) Profile comparison of the silicon master and replicated nanopillar and 3-dimensional surface profiles of (e) silicon master and (f) replicated nanopillar pattern.

(2000 Flood, Dymax). To prevent the replica adhering to the mold, a SAM coating was applied for each of the replication processes. Since a SAM treatment directly on the polymer surface can damage the polymeric structure, a thin film layer of $10 \mathrm{~nm} \mathrm{SiO}_{2}$ was deposited on the surface of the polymeric replica by e-beam evaporation at $6 \times 10^{-6}$ torr and a $0.3 \AA / \mathrm{s}$ deposition rate, and it was subsequently dipped into a $2 \%$ solution of dimethyldichlorosilane dissolved in octamethylcyclooctasilane (Repel-Silane ES, GE Healthcare
Co., Ltd., USA). After 20 minutes, the sample was rinsed with deionized (DI) water and dried at $30^{\circ} \mathrm{C}$.

\section{Results and Discussion}

Figures 2(a) 2(c) show the scanning electron microscope (SEM) images of the silicon master, polymeric first replica of the silicon master, and the nanopillar pattern replicated from the polymeric master. A field emission-SEM (SIGMA, Carl 


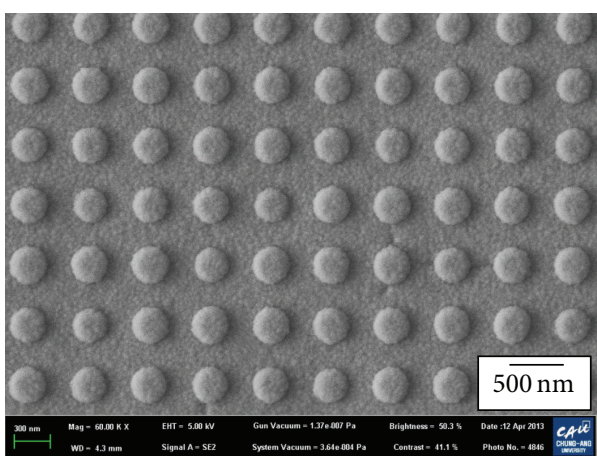

(a)

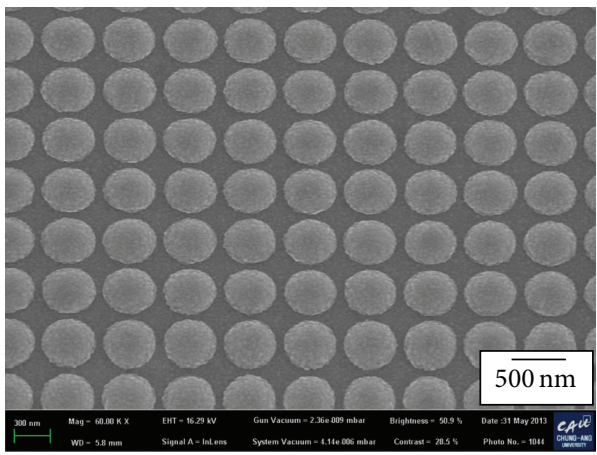

(c)

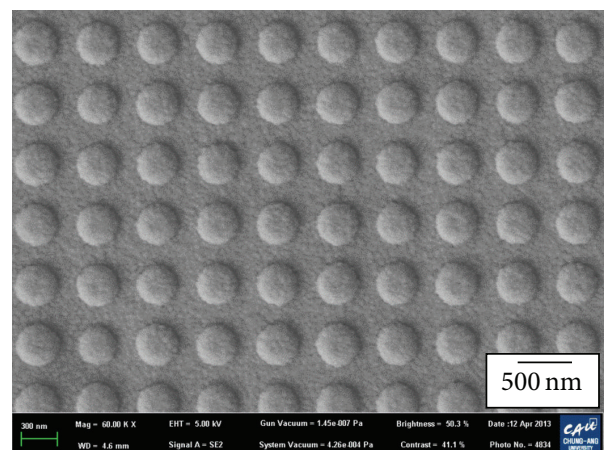

(b)

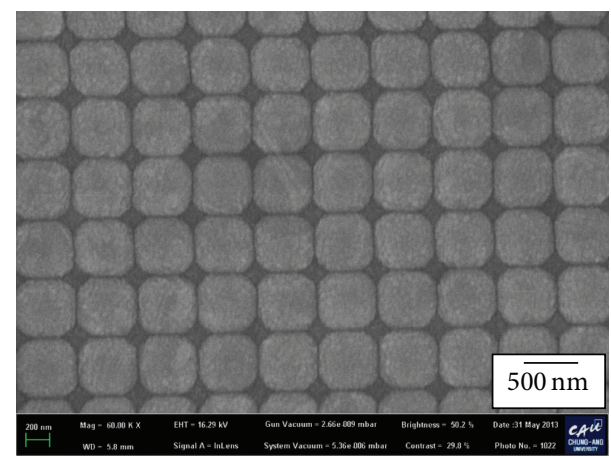

(d)

FIGURE 3: SEM images of the fabricated nanodome master with deposited $\mathrm{SiO}_{2}$ thicknesses of (a) 50, (b) 100, (c) 150, and (d) $200 \mathrm{~nm}$; EHT of $5 \mathrm{kV}$ was used for (a) and (b) and $16.29 \mathrm{kV}$ was used for (c) and (d).

Zeiss Co., Ltd., Germany) was used for SEM measurement with an EHT of $5.00 \mathrm{kV}$ (for Figure 2(a)) and an EHT of $16.29 \mathrm{kV}$ (for Figures 2(b) and 2(c)). Figures 2(e) and 2(f) show the 3-dimensional surface profiles of the silicon master and the replicated nanopillar obtained by atomic force microscope (AFM) measurements. The AFM measurement was conducted by XE-100 (Park Systems Co., Ltd., Korea) with a noncontact scanning mode and a speed of $0.3 \mathrm{~Hz}$ using a noncontact AFM tip (PPP-NCHR-50, Park Systems Co., Ltd., Korea). Figure 2(d) shows the comparison of AFM measured cross-sectional surface profiles of the silicon master and the replicated nanopillar. According to Figure 2(d), the pattern sizes on the replica were slightly smaller than those on the silicon master wafer due to shrinkage of the imprinting material during polymerization and the thin film of $\mathrm{SiO}_{2}$ (thickness of $10 \mathrm{~nm}$ ) deposited for SAM treatment.

Subsequently, the $\mathrm{SiO}_{2}$ deposition process was performed on the replicated nanopillar structure to form a dome-shaped structure with 4 different thicknesses $(50,100,150$, and $200 \mathrm{~nm}$ ) using e-beam evaporation. Figure 3 shows the SEM images of the prepared nanodome master (Figure 1(c)). To evaluate the detailed geometrical properties of the nanodome master, the prepared nanodome samples were measured by AFM. Figure 4 shows the measured profiles of the nanodome master. For deposited $\mathrm{SiO}_{2}$ thicknesses of $0,50,100$, and $150 \mathrm{~nm}$, the diameters of the nanodome base were 246, 300, 353 , and $436 \mathrm{~nm}$, respectively (Figure 4 ). Since the pitch of the pattern was $500 \mathrm{~nm}$, the separation distance of the nanodome

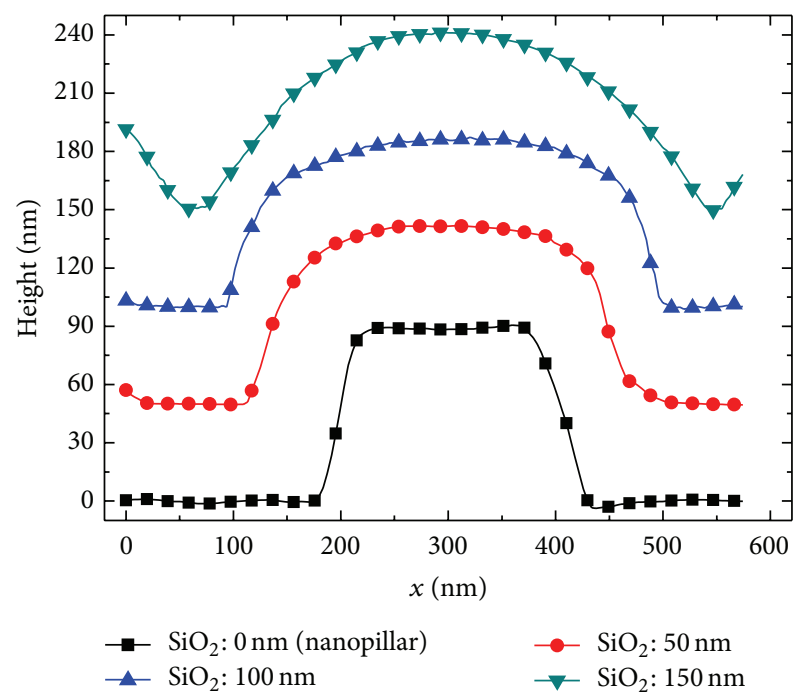

FIGURE 4: AFM of the nanodome master with different $\mathrm{SiO}_{2}$ deposition thicknesses.

decreased when the thickness of the $\mathrm{SiO}_{2}$ layer increased. As shown in Figure 3(d), when the separation distance is smaller than 0 , the structures can evolve into a square shape, which can act as $\mathrm{SiO}_{2}$ thickness limitation for forming dome structures. Although the initial pillar pattern has a rectangular cross-sectional shape (Figure 4, black line), it is found 


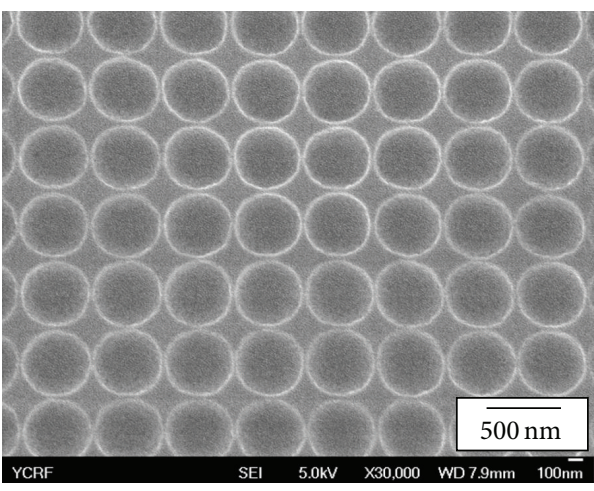

(a)

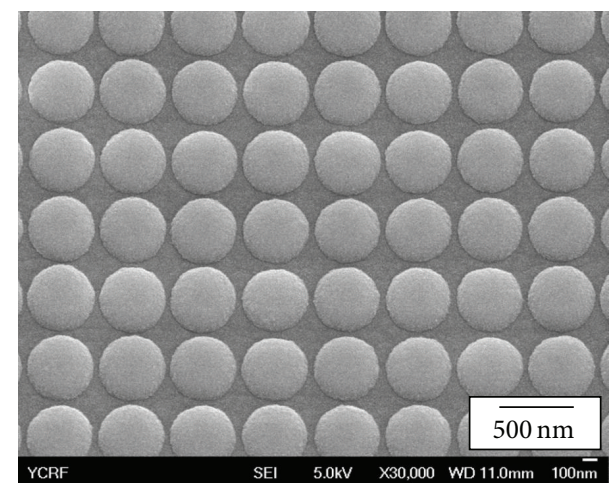

(b)

FIGURE 5: SEM images of (a) fabricated nanodome mold with a dome-shaped cavity array and (b) replicated polymeric nanodome on a glass substrate; EHV of $5 \mathrm{kV}$ was used for both measurements.

that the edge of the pattern was rounded as increasing the deposition thickness of the $\mathrm{SiO}_{2}$. At the $\mathrm{SiO}_{2}$ layer thickness of $150 \mathrm{~nm}$, we can confirm that the cross-sectional profile of the structure was well matched with dome shape, which can provide gradual refractive index change and good antireflection characteristics. $150 \mathrm{~nm}$ was selected for the thickness of the $\mathrm{SiO}_{2}$ layer to ensure an improved dome shape.

The fabricated nanodome master was then replicated on a PET film to create a nanodome mold by the UV imprinting process. For the next UV imprinting process, the SAM was coated with the same manner as the previous replication processes with a polymeric mold. Figure 5(a) shows the prepared nanodome mold with a $10 \mathrm{~nm} \mathrm{SiO}_{2}$ layer and SAM. The nanodome mold was employed to fabricate nanodomes on a glass substrate by the UV imprinting process. The adhesion promoter treatment was performed on the glass substrate. Figure 5(b) shows the fabricated nanodome array.

To evaluate the antireflective performance of the fabricated nanodome array, the transmission spectra through the sample were measured using a spectrophotometer (UV-670 UV-Vis, Jasco Inc., USA) in the wavelength range of 350 to $850 \mathrm{~nm}$. Figure 6 shows the measured transmittance of the flat and nanodome featured glass substrates. While the flat glass substrate showed $88-89 \%$ transmittance in the visible range, the transmittance of the nanodome featured glass substrate increased to $91-92 \%$. Since the antireflective nanodome structure was applied to only one side of the glass substrate, the transmittance can be improved up to $\sim 95 \%$ when the developed structure is applied to both sides of the glass substrate, because additional antireflection effects can be obtained from the nanodome structured backside surface [7].

\section{Conclusions}

In this paper, we demonstrated a fabrication method for antireflective nanodome patterns on a glass substrate. A series of UV imprinting processes were performed. First, a polymeric nanopillar array was fabricated by a UV replication process using a silicon nanomaster produced by $\mathrm{KrF}$ lithography and reactive ion etching processes. Subsequently,

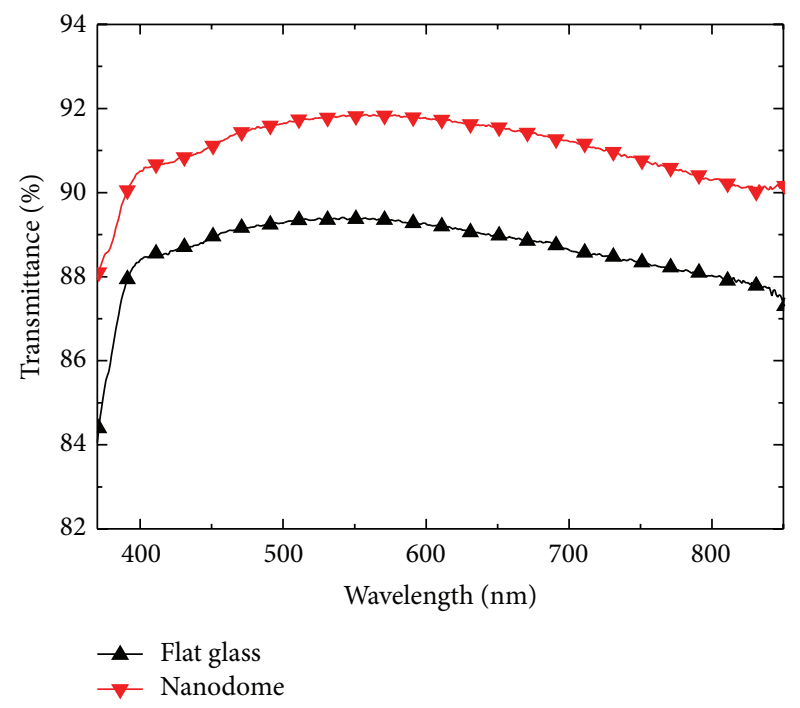

FIGURE 6: Transmittance of the flat and nanodome featured glass substrates.

a $150 \mathrm{~nm} \mathrm{SiO}$ 2 layer was deposited on the polymeric nanopillar array to form a nanodome master, and then it was replicated once more to create a nanodome mold. Finally, the antireflective nanodome structure was fabricated using the prepared mold. The SAM coating was applied for each of the replication processes. The dome shape was controlled by the thickness of the $\mathrm{SiO}_{2}$ layer. The optical transmittance of the fabricated sample was measured to evaluate the antireflective performance. The transmittance increased by approximately $3 \%$ compared to the bare glass substrate with a single-sided feature sample. Although we deposited $\mathrm{SiO}_{2}$ layer on the nanoimprinted pillar array to examine the effects of shape change while preserving the expensive silicon master pattern, the proposed nanodome master fabrication method by the deposition of $\mathrm{SiO}_{2}$ layer on the pillar pattern can be directly applied to the photolithographed silicon pillar array. We believe that the nanodome master fabrication process using photolithographed silicon pillar array and physical vapor 
deposited $\mathrm{SiO}_{2}$ layer can provide a uniform and defect-free nanodome master pattern in large area (up to $300 \mathrm{~mm}$ ), because the photolithography and physical deposition processes are the industrially well-established techniques. This fabrication method can be used to create antireflective surfaces for various applications such as solar cells, illumination, and imaging optics.

\section{Conflict of Interests}

The authors declare that there is no conflict of interests regarding the publication of this paper.

\section{Acknowledgments}

This work was supported by the Human Resources Program in Energy Technology of the Korea Institute of Energy Technology Evaluation and Planning (KETEP) granted financial resource from the Ministry of Trade, Industry \& Energy, Republic of Korea (no. 20134030200350) and was also supported by the Chung-Ang University Research Scholarship Grants in 2013.

\section{References}

[1] H. K. Raut, V. A. Ganesh, A. S. Nair, and S. Ramakrishna, "Anti-reflective coatings: a critical, in-depth review," Energy \& Environmental Science, vol. 4, pp. 3779-3804, 2011.

[2] S. Hong, B. Bae, K. Han, E. Hong, H. Lee, and K. Choi, "Imprinted moth-eye antireflection patterns on glass substrate," Electronic Materials Letters, vol. 5, no. 1, pp. 39-42, 2009.

[3] J. W. Leem, D. H. Joo, and J. S. Yu, "Biomimetic parabolashaped AZO subwavelength grating structures for efficient antireflection of Si-based solar cells," Solar Energy Materials \& Solar Cells, vol. 95, no. 8, pp. 2221-2227, 2011.

[4] K. M. A. Sobahan, Y. J. Park, J. J. Kim, and C. K. Hwangbo, "Nanostructured porous $\mathrm{SiO}_{2}$ films for antireflection coatings," Optics Communications, vol. 284, no. 3, pp. 873-876, 2011.

[5] M. Sun, A. Liang, Y. Zheng, G. S. Watson, and J. A. Watson, "A study of the anti-reflection efficiency of natural nano-arrays of varying sizes," Bioinspiration \& Biomimetics, vol. 6, no. 2, Article ID 026003, 2011.

[6] Y. M. Song, H. J. Choi, J. S. Yu, and Y. T. Lee, "Design of highly transparent glasses with broadband antireflective subwavelength structures," Optics Express, vol. 18, no. 12, pp. 13063-13071, 2010.

[7] K. Han, J. Shin, and H. Lee, "Enhanced transmittance of glass plates for solar cells using nano-imprint lithography," Solar Energy Materials \& Solar Cells, vol. 94, no. 3, pp. 583-587, 2010.

[8] W. C. Luk, K. M. Yeung, K. C. Tam et al., "Enhanced conversion efficiency of polymeric photovoltaic cell by nanostructured antireflection coating," Organic Electronics, vol. 12, no. 4, pp. 557-561, 2011.

[9] T. Søndergaard, J. Gadegaard, P. K. Kristensen, T. K. Jensen, T. G. Pedersen, and K. Pedersen, "Guidelines for 1D-periodic surface microstructures for antireflective lenses," Optics Express, vol. 18, no. 25, pp. 26245-26258, 2010.

[10] S. Kim, W. Zhang, and B. T. Cunningham, "Coupling discrete metal nanoparticles to photonic crystal surface resonant modes and application to Raman spectroscopy," Optics Express, vol. 18, no. 5, pp. 4300-4309, 2010.

[11] Y. Han, E. Byeon, J. Kim, and S. Kim, "A simulation model considering sidewall deposition for the precise prediction of the performance of label-free photonic-crystal biosensors," Journal of Nano Science and Nano Technology, vol. 12, no. 7, pp. 54295434, 2012.

[12] S.-J. Liu and W.-A. Chen, "Nanofeatured anti-reflective films manufactured using hot roller imprinting and self-assembly nanosphere lithography," Optics \& Laser Technology, vol. 48, pp. 226-234, 2013.

[13] C. J. Choi, Z. Xu, H. Wu, G. L. Liu, and B. T. Cunningham, "Surface-enhanced Raman nanodomes," Nanotechnology, vol. 21, no. 41, Article ID 415301, 2010. 

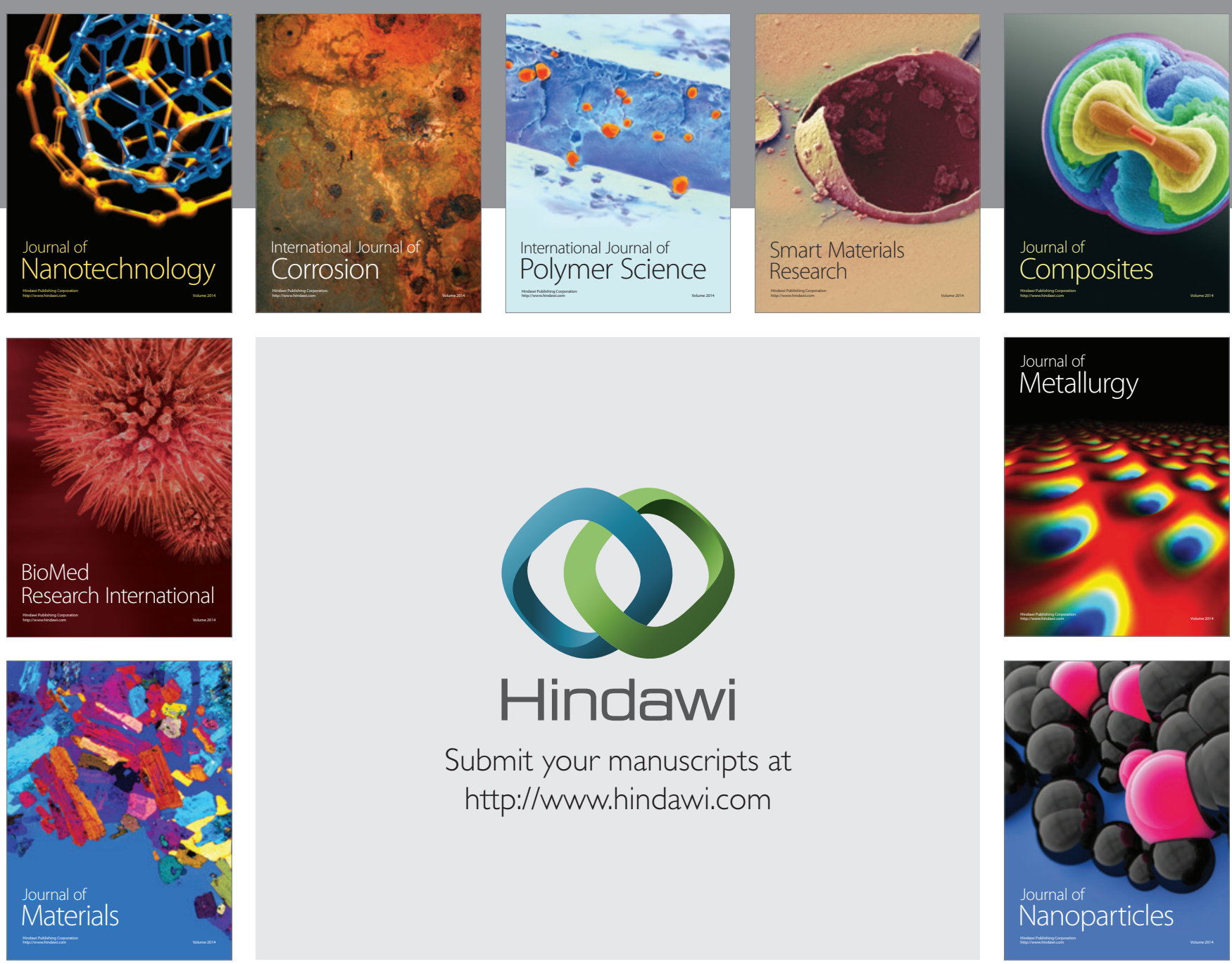

Submit your manuscripts at http://www.hindawi.com
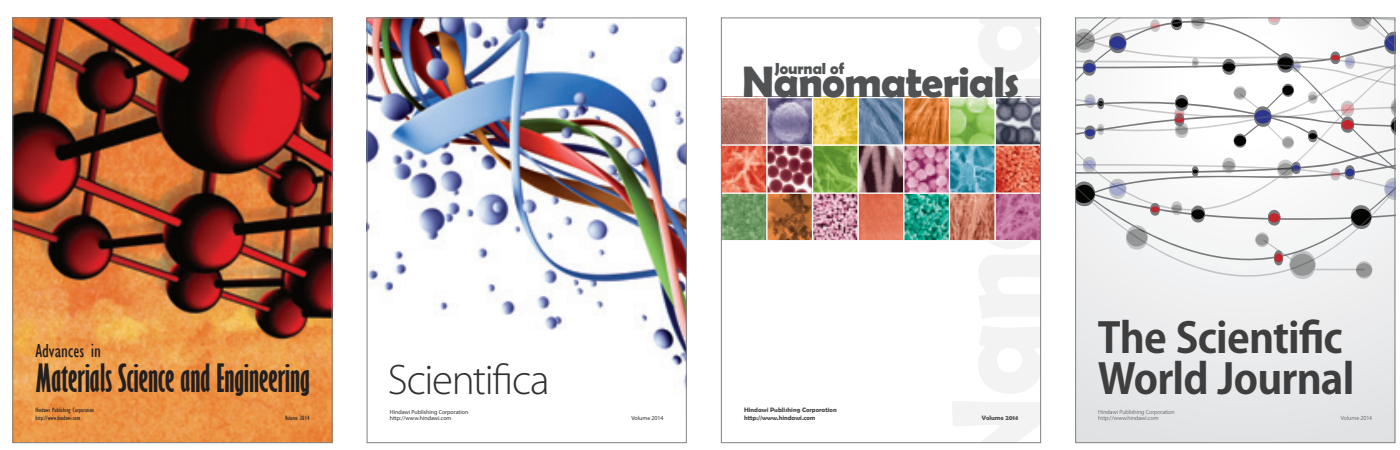

\section{The Scientific World Journal}
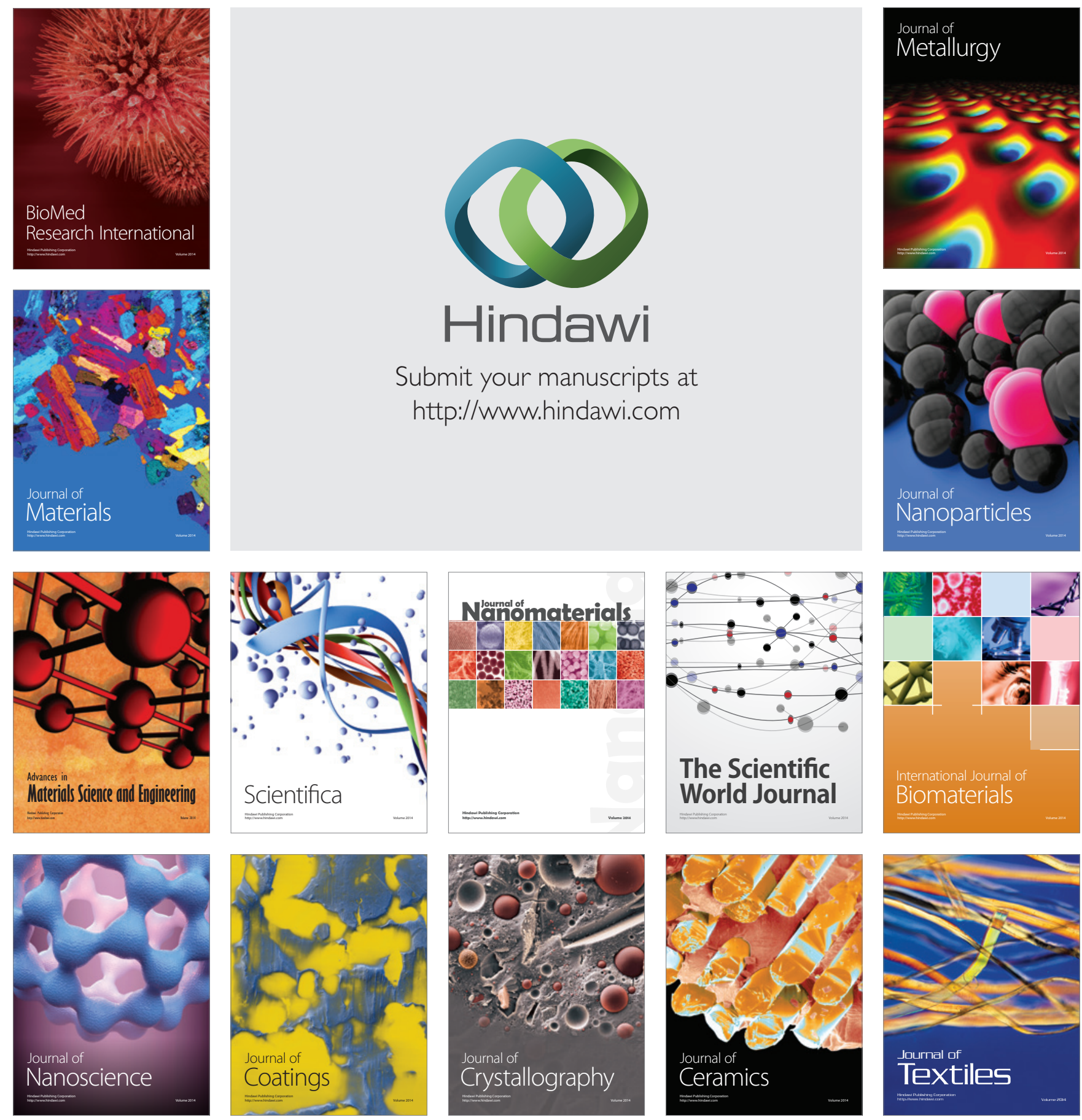